\title{
NEUE THERAPIEN BEI PÄDIATRISCHEN EPILEPSIEN
}

\section{Einleitung}

Trotz der Entwicklung zahlreicher neuer Medikamente in den letzten Jahrzehnten hat sich die Zahl der Patienten mit pharmakorefraktären Epilepsien kaum verändert. Das zunehmend bessere Verständnis der Ätiologie von Epilepsien, einschließlich genetischer und struktureller Ursachen, ermöglicht es, bei einigen Patienten spezifische molekulare Targets für Therapien zu identifizieren, die über die Anfallshemmung hinausgehen und die Behandlung der Ursache der Epilepsie ermöglichen. Die grösste Herausforderung wird es weiterhin sein, die Ursachen der Epilepsie frühzeitig zu identifizieren und so dem Patienten die beste Therapieoption zu ermöglichen.

Die Rationale für die Auswahl von Antiepileptika (AED) im Kindes- und Jugendalter sollte in Abhängigkeit von deren Wirkmechanismen (Abbildung 1) so individuell wie möglich gestaltet werden. Dabei werden sowohl altersspezifische Aspekte der Pharmakokinetik und -dynamik als auch anfallsspezifische, ggf. Epilepsiesyndrom spezifische Überlegungen berücksichtigt, im Idealfall auch Überlegungen zur molekulargenetischen und damit funktionellen Ursache der Epilepsie auf Zellebene. Letzteres öffnet Perspektiven für eine individualisierte Therapie, die neben den Anfällen z.B. auch eine assoziierte Entwicklungsstörung positiv beeinflussen kann.
Das Ziel jeder antiepileptischen oder anfallspräventiven Behandlung ist in erster Linie, weitere Anfälle zu verhindern. Zusätzlich zu einer anfallspräventiven Wirkung möchte man auch eine frühe sog. antiepileptogene Wirkung erzielen, um eine Chronifizierung der Epilepsie zu verhindern. Obwohl dieser Effekt für einige AED (antiepileptic drugs) im Tiermodell nachgewiesen wurde, konnte dies beim Menschen bisher nicht repliziert werden. Neuroprotektion ist ein weiterer Effekt, den AED haben sollten, um einen negativen Einfluss von wiederholten Anfällen oder kontinuierlicher epileptischer Aktivität auf die Entwicklung und Kognition zu verhindern. Der Nachweis dieses Therapieziels bleibt von vielen Confoundern abhängig und ist daher schwierig.

Die Auswahl der besten bzw. möglichst massgeschneiderten Therapie kann auf 3 Stufen erfolgen:

- Anfallsspezifische Therapie (Auswahl des AED empirisch nach Anfallstyp, fokal, generalisiert oder unbekannt)

- Epilepsiesyndrom-spezifische Therapie

- Genspezifische Therapie (individualisiert bzw. personalisierter Therapiezugang)

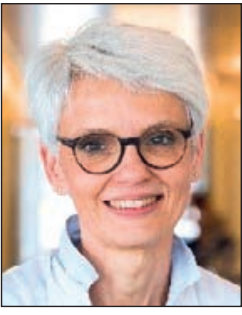

Judith Kröll

https://doi.org/ 10.35190/d2021.3.3

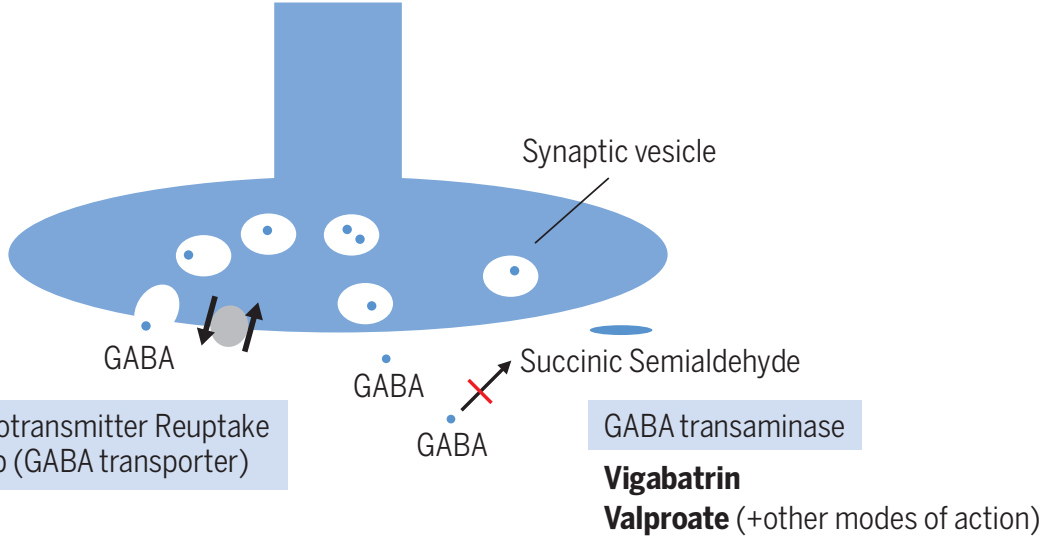

Tiagabine Neurotransmitter Reuptake pump (GABA transporter)

Valproate (+other modes of action)

Barbiturate Benzodiazepine, Topiramated (+ other modes of action)

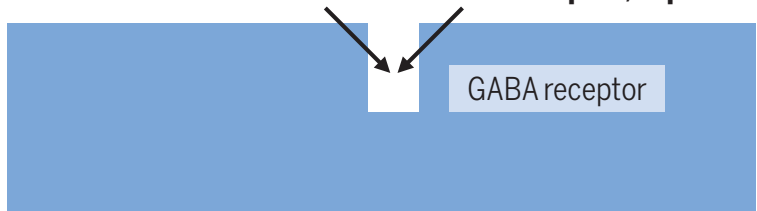




\section{Fortbildung}

\section{EXCITATORY NEUROTRANSMITTER SYSTEM and antiepileptic drugs}

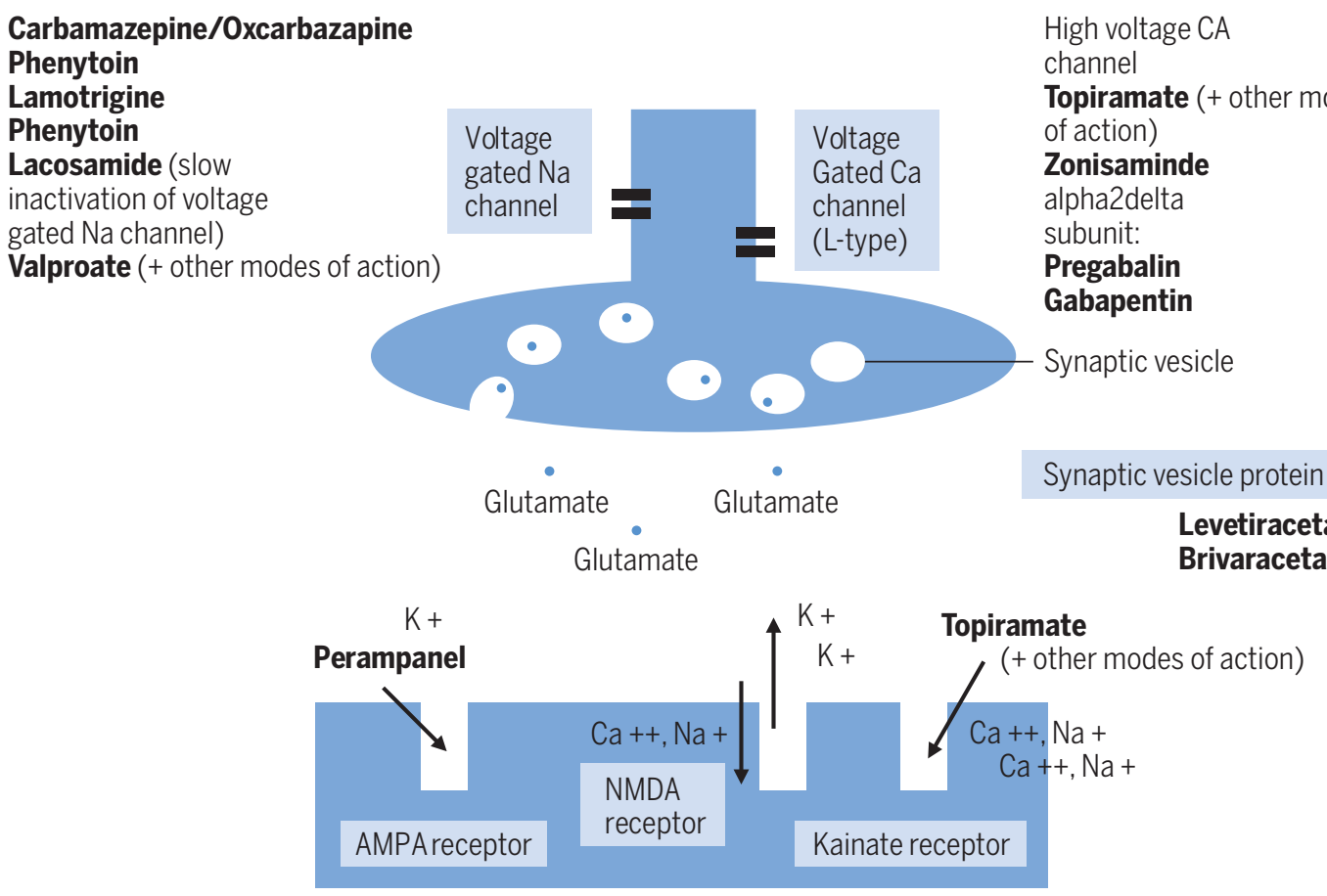

Abbildung 1: Mögliche Wirkmechanismen antiepileptischer/anfallspräventiver Medikamente ${ }^{15)}$

\section{A) Neue Epilepsiesyndrom-spezifische Therapien}

2020 bzw. 2021 wurden zwei sog. Orphan Drugs zur Behandlung von seltenen pädiatrischen Epilepsiesyndromen in der Schweiz und/oder der EU zugelassen:

\section{Cannabidiol}

Ein anfallspräventiver Effekt des nicht-psychoaktiven Cannabinoids Cannabidiol (CBD) wird bereits seit vielen Jahrzehnten beschrieben. Tierexperimentell konnten zwischenzeitlich verschiedene molekulare Targets identifiziert werden, über die CBD die neuronale Hyperexzitabilität beeinflusst. Ein möglicher anfallspräventiver Effekt der Substanz beruht u.a. darauf, dass der erregende Neurotransmitter Glutamat synaptisch weniger ausgeschüttet wird (Antagonismus an einem G protein-gekoppelten-Rezeptor) ${ }^{1)}$.

Nach Durchführung von mehreren doppelblinden und Placebo-kontrollierten Studien (RCTs) ist das Cannabidiol-Monopräparat Epidyolex ${ }^{\circledR}$ seit dem 10.2.2021 als Zusatztherapie bei epileptischen Anfällen bei Patienten mit Lennox-Gastaut-Syndrom (LGS) oder dem Dravet-Syndrom (DS) ab dem Alter von 2 Jahren in der Schweiz zugelassen und seit August 2021 erhältlich. Beide Epilepsiesyndrome sind sehr selten und die Anfälle sind bei den meisten Betroffenen pharmakorefraktär.

Das Präparat ist eine ölige Lösung (100 mg/ml). CBD ist lipophil und zeigt bei oraler Einnahme einen hohen first-pass-Metabolismus in der Leber mit einer Bioverfügbarkeit von ca. $6 \%$. Es interagiert mit mehreren Cytochrom P450-Enzymen (CYP) und hat eine Halbwertszeit von 18-32 Stunden ${ }^{2)}$. Die Interaktion mit CYP-Enzymen ist bei Kombinationstherapien zu beachten, insbesondere kann der aktive Metabolit des Benzodiazepins Clobazam um bis zu 50\% ansteigen, so dass die Dosis für eine Sedierung reduziert werden muss.

In einer doppelblinden Placebo-kontrollierten Studie, die 120 Kinder, Jugendliche und junge Erwachsene (Durchschnittsalter 9.8 Jahre, Range 2.3 bis 18.4 Jahre) mit DS eingeschlossen hat, zeigten in der CBDGruppe 43\% eine mindestens 50\%-ige Reduktion konvulsiver Anfälle vs. $27 \%$ in der Placebo-Gruppe, $3 \%$ wurden anfallsfrei. Unter Berücksichtigung der Grunderkrankung, ist dieses Ergebnis für die Betroffenen als positiv zu werten. Die häufigsten Nebenwirkungen waren Müdigkeit und Durchfall ${ }^{3}$.

Das Dravet-Syndrom (DS) ist ein seltenes pädiatrisches Epilepsiesyndrom, das 1/15.700 bis $1 / 40.000$ Kinder betrifft ${ }^{4}$. Beim klassischen DS treten die ersten Anfälle typischerweise zwischen dem 4. und 8. Lebensmonat auf. Sie sind klonisch, generalisiert oder unilateral und es besteht ein hohes Risiko für prolongierte Anfälle oder Status epilepticus. Die Anfälle werden oft durch (leichtes) Fieber ausgelöst. Im Alter von 2 bis 5 Jahren können myoklonische Anfälle, (atypische) Absencen und fokale Anfälle auftreten. Gleichzeitig verschlechtern sich die motorischen Funktionen und eine kognitive Einschränkung wird deutlich.

De novo-Mutationen im SCN1A-Gen werden bei 75-85\% der Patienten mit DS nachgewiesen. 
Das SCN1A-Gen kodiert die $\alpha$-Untereinheit des wichtigsten spannungsabhängigen Typ-I-Natriumkanals (NaV1.1) im Gehirn, der spezifisch in GABA-ergen, inhibitorischen Interneuronen lokalisiert ist Krampfanfälle bei DS sind refraktär gegenüber konventionellen Antikonvulsiva. Die Behandlung des DS kann daher lediglich auf die Reduktion der Anfallslast abzielen, insbesondere von prolongierten Anfällen und Status epilepticus, auf die Vermeidung negativer Nebenwirkungen der antikonvulsiven («Über»-) Behandlung, die Verringerung des SUDEP (plötzlicher Tod bei Epilepsie) -Risikos und die Verbesserung der Komorbiditäten.

Bei Patienten mit LGS konnte in zwei randomisierten und kontrollierten Studien mit add-on CBD (10 und 20 mg/kg/d während 14 Wochen im Vergleich mit Placebo) gezeigt werden, dass sich die Zahl der Sturzanfälle (tonisch, atonisch oder unbekannt) reduzierte. Die mittlere prozentuale Reduktion der Anfallshäufigkeit während des Behandlungszeitraums betrug 41,9 $\%$ in der 20-mg-CBD-Gruppe, 37,2 \% in der 10-mgCBD-Gruppe und $17,2 \%$ in der Placebo-Gruppe ( $p=0,005$ für die 20-mg-Cannabidiol-Gruppe) ${ }^{5,6)}$.

Das Lennox-Gastaut-Syndrom (LGS) ist eine der schwersten epileptischen und entwicklungsbedingten Enzephalopathien. Es macht $1-10 \%$ aller Epilepsien im Kindesalter aus. Es gibt mehrere gleichzeitig auftretende Anfallstypen einschließlich plötzlicher Sturzanfälle (kurze tonische oder atonische Anfälle) mit hohem Verletzungsrisiko, atypische Absencen, die in einen nicht konvulsiven Status epilepticus übergehen können, und andere Anfallstypen, die fokal, generalisiert, tonisch-klonisch oder einseitig klonisch sind. Der vorherrschende Anfallstyp kann sich im Laufe der Zeit ändern. Das LGS ist immer mit einem kognitiven Abbau, oft schweren Verhaltensstörungen und Medikamentenresistenz assoziiert. Erkrankungsbeginn liegt in der Regel vor dem Alter von 8 Jahren mit einem Höhepunkt zwischen 3 und 5 Jahren.

Die Ätiologie ist sehr heterogen und umfasst strukturelle Anomalien des Gehirns, prä, perioder postnatale hypoxisch-ischämische Ereignisse, Infektionen, metabolische oder toxische Hirnverletzungen oder genetische Anomalien. Etwa 40\% der Patienten haben eine Vorgeschichte von infantilen Spasmen ${ }^{7}$.

In einer Zwischenanalyse zur langfristigen Wirksamkeit und Verträglichkeit von $\mathrm{CBD}$, das Patienten mit LGS $(N=152)$, DS ( $N=58)$ oder anderen therapierefraktären Epilepsien ( $N=455)$ in einem erweiterten Zugangsprogramm erhielten, zeigte sich, dass die add-on Therapie mit CBD eine sichere und wirksame langfristige Behandlungsoption für diese Patientengruppen darstellt. Die mediane Behandlungsdauer betrug 78,3 (Range 4,1-146,4) Wochen. Zwischen Woche 12 und 96 lag die mediane add-on CBD-Dosis zwischen 21 und $25 \mathrm{mg} / \mathrm{kg} / \mathrm{Tag}$. Nach 12 Wochen reduzierte diese add-on Gabe von CBD die monatlichen motorischen Anfälle im Median um $50 \%$ und die Gesamtzahl der Anfälle um $44 \%$, wobei die Anfallsreduktion über 96 Wochen hinweg konstant blieb. 20\% der LGS/ DS-Patienten setzen CBD wieder ab hauptsächlich wegen mangelnder Wirksamkeit. $5,6,8)$

\section{Fenfluramin}

Fenfluramin ist ein Wirkstoff aus der Gruppe der Amphetamine, der die Ausschüttung des Neurotransmitters Serotonin erhöht. Ursprünglich wurde Fenfluramin als Appetitzügler eingesetzt. Wegen schwerwiegender kardiovaskulärer Nebenwirkungen (pulmonale arterielle Hypertonie, Verdickung der Herzklappen) wurde die Substanz 1997 vom Markt genommen. Während der Zeit der Zulassung als Appetitzügler wurde bei Epilepsie-Patienten die antiepileptische Wirkung beobachtet.

Das Medikament Fintepla ${ }^{\circledR}$ enthält eine orale Fenfluramin-Lösung (2,2 mg/ml). Das Medikament wurde am 18.12.2020 als sog. Orphan Drug zur Behandlung des Dravet-Syndroms als add-on Therapie ab dem Alter von 2 Jahren in der EU zugelassen.

In zwei RCTs konnte gezeigt werden, dass Fenfluramin die Häufigkeit von Anfällen bei Kindern und jungen Erwachsenen mit Dravet-Syndrom, die mindestens ein anderes Epilepsie-Medikament einnehmen, verringert. Bei insgesamt 206 Patienten mit Dravet-Syndrom (Studie 1 mit 119 Patienten und Studie 2 mit 87 Patienten) wurde als Hauptindikator für die Wirksamkeit die Änderung der monatlichen Anzahl von Anfällen beurteilt. In der ersten Studie reduzierte sich in der Gruppe mit einer täglichen Fenfluramin-Dosierung von $0,7 \mathrm{mg} / \mathrm{kg}$ die mittlere monatliche Anfallshäufigkeit der Baseline-Phase in der Erhaltungsphase signifikant um 62,3\% (95\%-Cl [47,7-72,8]; $p<0,0001)$ im Vergleich zu Placebo. In der Gruppe mit einer niedrigen Dosierung von $0,2 \mathrm{mg} / \mathrm{kg}$ Fenfluramin täglich wurde eine mittlere Reduktion um 32,4\% (95\%-Cl [6,2-52,3]; $p=0,0209)$ erreicht.

Bei $73 \%$ der mit Fenfluramin behandelten Patienten und bei $10 \%$ der mit Placebo behandelten Patienten ging die Anzahl der Anfälle pro Monat um mindestens $50 \%$ zurück ${ }^{9}$.

In der zweiten Studie erhielten die Patienten zusätzlich Stiripentol (eine weitere Orphan drug zur Behandlung des DS) und mindestens ein weiteres Antikonvulsivum. In der Gruppe, die zusätzlich zu Stiripentol 0,4 mg Fenfluarmin/kg/Tag erhielt, reduzierte sich die mittlere monatliche Anfallshäufigkeit in der Erhaltungsphase um 54,0\% (95\% Cl [35,6-67,2]; $\mathrm{p}<0,001)$ im Vergleich zu Placebo ${ }^{10}$.

Unter Sicherheitsaspekten sind vor Beginn der Behandlung sowie auch im weiteren Verlauf regelmäs- 


\section{Fortbildung}

sige echokardiographische Untersuchungen vorgeschrieben. Die Dosierung des Antikonvulsivums ist deutlich niedriger verglichen mit dem Einsatz als Appetitzügler. In den oben aufgeführten Studien wurden keine kardiologischen Nebenwirkungen beobachtet.

\section{B) Individualisierte molekulare} und Genspezifische Therapien

Individualisierte molekulare und genspezifische Therapien ermöglichen eine personalisierte Medizin. Mit der Identifikation von sog. Epilepsiegenen in den letzten Jahrzenten konnten zunehmend die molekularen Mechanismen entschlüsselt werden, die dem klinischen Erscheinungsbild zugrunde liegen. So verbesserte sich nicht nur das Verständnis dieser Epilepsien, sondern es werden auch zielgerichtete Therapien möglich.

Drei Arten von zielgerichteten Therapien können in der Behandlung von Epilepsien unterschieden werden: erstens Substitutionstherapien, zweitens Therapien, die Signalwege blockieren, und drittens Therapien, die die Leitfähigkeit von lonenkanälen normalisieren ${ }^{11)}$.

Substitutionstherapien spielen bei den metabolischen Epilepsien und Enzephalopathien eine Rolle. Beispiele sind der Einsatz der ketogenen Diät bei dem sog. Glukose-Transporter-Defekt-Typ 1 oder die Behandlung der Pyridoxin und Pyridoxal-phosphat-abhängigen Epilepsien (weitere Erkrankungen s. auch Tabelle). Die frühe Diagnose und gezielte Substitutionsbehandlung ist mehr als nur eine Anfallsbehandlung, sondern sie soll auch die Entwicklungsprognose der betroffenen Kinder verbessern.

Für die spät-infantile neuronale Zeroidlipofuszinose Typ 2 (CLN2, Beginn mit 2-4 Jahren, Mutation im TPP1 Gen), einer lysosomalen Erkrankung, die auch als kindliche Demenz bezeichnet wird und die typischerweise im Kleinkindalter mit Anfällen beginnt und mit einer raschen kognitiven und motorischen Entwicklungsregression einhergeht, wurde eine Enzymersatztherapie entwickelt. Cerliponase Alpha (Brineura ${ }^{\circledR}$ ), ein rekombinantes Proenzym der menschlichen Tripeptidyltranferase 1 wird als intraventrikuläre Infusion alle 2 Wochen appliziert. Bei 23 behandelten Kindern im Alter von 3-16 Jahren konnte gezeigt werden, dass durch die Behandlung die Verschlechterung der motorischen und Sprachfunktionen verlangsamt wird im Vergleich mit historischen Kontrollen. Die Behandlung ist spezialisierten Kliniken vorbehalten ${ }^{12}$.

Beispiel für die Blockade eines Signalwegs ist der Einsatz von Everolimus zur Blockade des sog. mTOR-Pathway.

Der mTOR-Pathway spielt u.a. eine Schlüsselfunktion in der Hirnentwicklung. Seine Hyperexpression führt zu Störungen der Entwicklung des zerebralen Kortex bei Tuberöse Sklerose Komplex (TSC), aber auch bei fokalen kortikalen Dysplasien oder der Hemimegalenzephalie. Mittlerweile sind zahlreiche Mutationen in Genen identifiziert, die in diesem Signalweg involviert sind (MTOR, PIK3CA, TSC1, TSC2 und PTEN Gene sowie die GATOR1-Komplex-Gene DEPDC5, NPRL2 und NPRL3).

Bei therapierefraktären Anfällen bei TSC ist eine ätiologiebasierte Therapie bereits möglich. In der multizentrischen EXIST 3 Studie konnte gezeigt werden, dass die adjuvante Gabe des mTOR -Inhibitors Everolimus bei pädiatrischen TSC-Patienten mit therapierefraktären Anfällen zu einer anhaltenden Reduktion der Anfallshäufigkeit nach 1 Jahr führte und gut verträglich war ${ }^{13,14)}$.

\section{Normalisierung der Leitfähigkeit von lonenkanälen}

Bei den Epilepsien, die zu der Gruppe sog. Ionenkanalerkrankungen gehören, d.h. vor allem Natrium- und Kaliumkanalopathien, gibt es sowohl selbstlimitierende Epilepsien als auch epileptische Enzephalopathien mit katastrophalen Verläufen.

Spannungsabhängige Natriumkanäle vermitteln die Entstehung und Propagation von Aktionspotentialen. Viele konventionelle Antikonvulsiva antagonisieren diese Funktion. Von Mutationen, die eine Epilepsie verursachen, sind in absteigender Häufigkeit die Natriumkanalgene SCN1A, SCN2A und SCN8A betroffen Die Aktivierung von Kaliumkanälen reduziert die neuronale Erregbarkeit, so dass die Kaliumkanal-assoziierten Epilepsien meist mit loss-of-function-Mutationen assoziiert sind (s. Tabelle 1).

Exemplarisch werden nachfolgend SCN1A- und SCN2A-assoziierte Epilepsien vorgestellt. Heterozygote loss-of-function Mutationen im SCN1A-Gen finden sich beim sog. klassischen Dravet-Syndrom (s. oben). Es kommt zu einem Ungleichgewicht zwischen Inhibition und Exzitation, was zu einer erhöhten Anfallsbereitschaft führt. Da die loss-of-function Mutation vorwiegend die inhibitorischen Interneurone betrifft, kann man es sich gut vorstellen, dass die Gabe typischer Natriumkanalblocker wie Carbamazepin, Oxcarbazepin oder Phenytoin die Anfallskontrolle verschlechtert.

Das SCN2A-Gen kodiert ebenfalls für einen spannungsabhängigen Natriumkanal. SCN2A-assoziierte Erkrankungen kann man in 3 Gruppen unterteilen:

1) Frühkindliche epileptische Enzephalopathie (mit einem Auftreten der ersten Anfälle meist bereits innerhalb der ersten 3 Lebensmonate gefolgt von einer schweren Entwicklungsstörung.

2) Benigne/selbstlimitierende (familiäre) neonatale oder infantile Anfälle, charakterisiert durch Auftreten von Anfällen im Neonatalalter oder vor dem 12. Lebensmonat, die Epilepsie verläuft bis zum Alter von 2 Jahren selbstlimitierend ohne erkennbare Langzeitfolgen.

3) Autismus-Spektrum-Störung/intellektuelle Behinderung (ASD/ID) mit oder ohne Epilepsie. Die early-onset-Gruppe kann von dem Einsatz von Natriumkanalblockern profitieren. Es gibt Hinweise, dass 


\section{Fortbildung}

\begin{tabular}{|c|c|c|}
\hline Epilepsiesyndrom & Gen & Treatment \\
\hline $\begin{array}{l}\text { Dravet Syndrome, GEFS+ } \\
\text { BFNE, BFNIE } \\
\text { BFNE, BFNIE } \\
\text { BFNE } \\
\text { BFNE }\end{array}$ & $\begin{array}{l}\text { Veränderungen in Natriumkanälen } \\
\text { SCN1A } \\
\text { SCN2A } \\
\text { SCN8A } \\
\text { Veränderungen in Kaliumkanälen } \\
\text { KCNQ2 } \\
\text { KCNQ3 }\end{array}$ & $\begin{array}{l}\text { Vermeide Natriumkanalblocker: } \\
\text { CBZ, OXC, PHT, LTG } \\
\text { CBZ, OXC, PHT, (LTG) } \\
\text { CBZ, OXC, PHT, (LTG) } \\
\\
\text { CBZ, OXC, PHT, (LTG) } \\
\text { CBZ, OXC, PHT, (LTG) }\end{array}$ \\
\hline BFNIE & PRTT2 & CBZ, OXC, PHT, (LTG) \\
\hline $\begin{array}{l}\text { DEE, Epilepsy in infancy with migrating focal s. } \\
\text { DEE, Epilepsy in infancy with migrating focal s. } \\
\text { DEE } \\
\text { DEE, Epilepsy in infancy with migrating focal s. } \\
\text { DEE, Epilepsy in infancy with migrating focal s. }\end{array}$ & $\begin{array}{l}\text { Veränderungen in Natriumkanälen } \\
\text { SCN2A } \\
\text { SCN8A } \\
\text { Veränderungen in Kaliumkanälen } \\
\text { KCNQ2, KCNQ3 } \\
\text { KCNT1 } \\
\text { KCNT2 }\end{array}$ & $\begin{array}{l}\text { CBZ, OXC, PHT, LTG } \\
\text { CBZ, OXC, PHT, LTG } \\
\text { CBZ, OXC, PHT, LTG; Retigabin (LoF) } \\
\text { Quinidin (GoF), Brom } \\
\text { Quinidin (GoF), Brom }\end{array}$ \\
\hline $\begin{array}{l}\text { Idiopathisch generalisierte Epilepsie, } \\
\text { DEE, West-Syndrom } \\
\text { GEFS+, DEE } \\
\text { GEFS+, DEE }\end{array}$ & $\begin{array}{l}\text { Veränderungen in Kalziumkanälen } \\
\text { CACNA1A } \\
\text { HCN1 LoF } \\
\text { HCN1 GoF }\end{array}$ & $\begin{array}{l}\text { ETX, LTG (GoF) } \\
\text { LTG, GBP } \\
\text { Ketamin, Propofol }\end{array}$ \\
\hline $\begin{array}{l}\text { VPA-assoziiertes Leberversagen } \\
\text { Carbamazepin und Steven Johnson Syndrom }\end{array}$ & $\begin{array}{l}\text { POLG1 } \\
\text { HLAB*1502 }\end{array}$ & $\begin{array}{l}\text { kein Valproat } \\
\text { kein Carbamazepin /Oxcarbazepin }\end{array}$ \\
\hline $\begin{array}{l}\text { Glucose-Transporter } 1 \text { Defizienz } \\
\text { Pyridoxin-abhängige Epilepsie } \\
\text { Pyridoxal-5 Phospate abhängige Epilepsie } \\
\text { Folinsäure-responsive Anfälle } \\
\text { Cerebrales Kreatin Defizienz-Syndrom } 1 \\
\text { Cerebrales Kreatin Defizienz-Syndrom } 2 \\
\text { Cerebrales Kreatin Defizienz-Syndrom } 3 \\
\text { CAD Defizienz, Pyrimidin-Synthese-Defizienz } \\
\text { Molybdän-Kofaktor Mangel (MOCOD) } \\
\text { Spätinfantile Zeroidlipofuszinose CLN2 }\end{array}$ & $\begin{array}{l}\text { SLC2A1 } \\
\text { ALDH7A1, ALDH7A } \\
\text { PNP0 } \\
\text { FOLR1 } \\
\text { SLC6A8 } \\
\text { GAMT } \\
\text { AGAT } \\
\text { CAD } \\
\text { MOCS1 } \\
\text { TPP1 }\end{array}$ & $\begin{array}{l}\text { ketogene Diät (KD) } \\
\text { Pyridoxin } \\
\text { Pyridoxal-5 Phosphat } \\
\text { Folinsäure } \\
\text { Kreatin + L-Arginine and L-Glyzin } \\
\text { Kreatin } \\
\text { Kreatin } \\
\text { Uridin-Monophosphat } \\
\text { Cyclic pyranopterin monophosphate } \\
\text { Cerliponase Alpha }\end{array}$ \\
\hline $\begin{array}{l}\text { Familiäre fokale Epilepsie mit variablen Foci, } \\
\text { familiäre mesiale Temporallappenepilepsie, } \\
\text { West Syndrom }\end{array}$ & $\begin{array}{l}\text { DEPDC5, GATOR1 } \\
\text { Komplex-Untereinheit }\end{array}$ & $\begin{array}{l}\text { Rapamycin und Rapamycin-Derivate } \\
\text { (z.B. Everolimus, Sirolimus, } \\
\text { Temsirolimus, Ridaforolimus }\end{array}$ \\
\hline Familiäre fokale Epilepsie mit variablen Foci & $\begin{array}{l}\text { NPRL2 and 3, GATOR1 } \\
\text { Komplex-Untereinheit }\end{array}$ & Rapamycin und Rapamycin-Derivate \\
\hline $\begin{array}{l}\text { Tuberöse Sklerose Komplex, } \\
\text { fokale kortikale Dysplasie }\end{array}$ & TSC1, TSC2 & $\begin{array}{l}\text { Rapamycin und Rapamycin- } \\
\text { Derivate, VGB, KD }\end{array}$ \\
\hline
\end{tabular}

GEFS+: Generalized epilepsy with febrile seizures + BFNE: self-limiting (benign) neonatal epilepsy BFNIE: self-limiting (benign) neonatal infantile epilepsy

DEE: Developmental and epileptic encephalopathy

LoF: Loss of function Mutation

GoF: Gain of function Mutation

CBZ = Carbamazepin, ETX = Ethosuximid, GBP = Gabapentin, LTG = Lamotrigin, OXC = Oxcarbazepin, PHT = Phenytoin,

VPA = Valproat, $\mathrm{VGB}=$ Vigabatrin

Tabelle 1: Gen-spezifische Therapien bei Epilepsien ${ }^{15)}$

es sich hierbei um gain-of-function-Mutationen handelt. Bei den später auftretenden Epilepsien (Gruppe 3) handelt es sich eher um loss-of function-Mutationen, so dass AED wie Levetiracetam, Benzodiazepine und Valproat zu bevorzugen sind, die keine Natriumkanalblockade bewirken.

\section{Ausblick}

Neue Substanzen wie Eslicarbazepin ${ }^{16)}$ und Cenobamate ${ }^{17)}$ werden in Zukunft auch bei Kindern durch erweiterte Wirkmechanismen die Verträglichkeit verbessern, resp. auch therapeutische Optionen vergrössern. 


\section{Fortbildung}

Für monogenetische Epilepsien werden im Tiermodell sog. antisense oligonucleotide (ASO)-Therapien getestet. Bei SCN1A-loss-of-function, SCN8A-gain-of-function oder KCNT1-gain of function-Mutationen gibt es erste positive Ergebnisse aus Mausmodellen. Diese neuen Therapien zielen darauf ab, die lonenkanalfunktion wiederherzustellen, so dass nicht nur die Anfälle besser kontrolliert, sondern auch die Komorbiditäten wie z.B. die kognitive Entwicklungsstörung, positiv beeinflusst werden. Weiter gibt es auch erste ermutigende Ansätze für Gentherapien mittels Vektoren in einem Mausmodell mit Dravet-Syndrom ${ }^{18)}$, Angelman Syndrom ${ }^{19)}$ und anderen genetischen Epilepsien.

Zusammengefasst ist die Identifizierung der Ursache und damit der zugrundeliegenden Funktionsstörungen der Epilepsien nicht nur entscheidend für Diagnose und Prognose, sondern auch die Voraussetzung für einen möglichst individualisierten Therapieansatz.

Für das Literaturverzeichnis verweisen wir auf unsere Online Version des Artikels.

\section{Autoren}

Dr. med. Judith Kröll, Sozialpädiatrisches Zentrum Konstanz

Dr. med. Alexandre N. Datta, Abteilung Neuro- und Entwicklungspädiatrie, Universitätskinderspital beider Basel (UKBB), Basel und Consultant für die Firmen Neurocrine, Idorsia und Epilog und in Advisory Boards von Eisai und Idorsia

Interessenkonflikt: 01-2021 Teilnahme an der online Arbeitskreistagung «Ketogene Ernahrungstherapie» der Fa. Nutricia; Interessenkonflikt: 02-2021 Teilnahme an einem online Advisory Board GWPharmaceuticals. 